\title{
CONSTRUCTAL THEORY OF PLATE TECTONICS
}

\author{
S. QUÉRÉ \\ Geosciences, Utrecht University, The Netherlands.
}

\section{ABSTRACT}

Labeled 'the all-time breakthrough in geology', plate tectonics brought a revolution in Earth Science 40 years ago. This theory links movements of lithospheric plates to processes taking place deep inside the Earth. The assumption of rigid surface plates enables one to describe the surface kinematics in terms of two components: the horizontal divergence (ridge-trench system) and the radial vorticity (transform faults and plate spin). The horizontal divergence and the radial vorticity are directly related to the poloidal (vertical motion around a lateral axis) and toroidal (lateral motion around a vertical axis) scalar fields, respectively. Surprisingly, kinetic energy in present-day plate motions is partitioned approximately equally between horizontal divergence (poloidal) and radial vorticity (toroidal) parts. I work with a time-dependant 3D spherical convective code where plates are coupled dynamically to the mantle. This method, which ensures the retrieval (to within $\pm 10 \%$ ) of the main global observables at the Earth's surface (mean plate velocity, global heat flux and potential temperature under oceanic ridges), is unique and provides new opportunities to tackle questions such as the coupling between plates and the deeper mantle. I made experiments with different plate geometry on top of the 3D spherical code. When I plot the degree variance of the poloidal and toroidal components of the surface velocity field, only a geometry close the observed presentday geometry shows almost the same toroidal to poloidal ratio that exists at present-day. It suggests that only a geometry close to the one which is observed at present-day can reproduce an equipartition between the poloidal and toroidal components of plate motions. Further, O'Connell et al. (Toroidal-poloidal partitioning of lithospheric plate motions. Glacial Isostasy, Sea Level and Mantle Rheology, eds R. Sabadini \& K. Lambeck, Kluwer Academic Publishers: Dordrecht, 1991) presume that, since the toroidal velocities are not involved in the heat transfer, the convective process develops a dynamical structure reducing the loss of kinetic energy, so that the partitioning essentially depends on the plate distribution. The constructal theory is covariant (the shape is changing in relation to the flow) as is plate tectonics. Plate motion reconstructions show that the geometry and plate motions are evolving slowly and probably maintain a lowest relative toroidal power through time. The constructal theory can be used to explain why the present-day configuration of plates is optimal for the Earth's mantle flow dynamics as the surface plate motions are indeed representative of the large-scale convective flow in the mantle.

Keywords: plate tectonics, mantle convection, plate motion reconstruction, constructal theory.

\section{INTRODUCTION}

\subsection{Continental drift: the premises of plate tectonics theory}

In 1912, Alfred Wegener [1] proposed the continental drift theory to explain the observations of continental blocs that drift relatively to each other. Alfred Wegener founded his theory on the base of three main observations: (1) the complementary arrangement of the facing sides of South America and Africa, (2) the similarity of fossil patterns across continents and (3) the continuity of glaciers across continents. Unfortunately, the continental drift theory has been rejected by geophysicist at that time, as Wegener was not able to provide proper arguments to explain it.

\subsection{Plate tectonics theory: a kinematic theory...}

After Wegener's death (1930) and during the Second World War, the ocean floor cartography showed the existence of oceanic ridges (longitudinal crests) in the middle of oceans as well as trenches (oceanic depressions). These observations were explained by Arthur Holmes [2] who stated that ocean floor was created at oceanic ridges and returning back to the mantle in the trenches. This new theory of ocean floor spreading was then confirmed by boat campaigns which recorded paleomagnetic data. All these 
observations led Jason Morgan (1967) to present the theory of plate tectonics at the American Geophysical Union Congress: the lithosphere (the top rigid part of the Earth) is divided into several plates that rotate relatively to each other at the surface of the Earth (Fig. 1). Each plate motion is entirely described by its angular motion [3]. The same year, McKenzie and Parker proposed an equivalent theory based on seismological arguments [4]. Plate tectonics was first described as a kinematic theory.

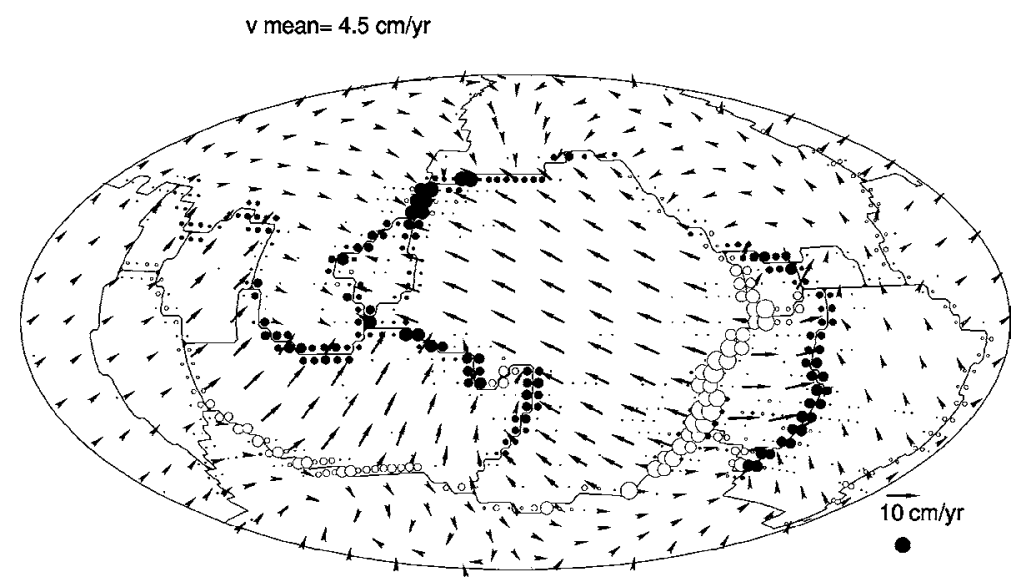

Figure 1: Present-day plate motions (NNR NUVEL1 model [5]). The black and white discs correspond to negative and positive vertical velocities, which refer to subduction zones and oceanic ridges, respectively.

1.3 Plate tectonics theory: ... and a dynamical theory

Around at the same period raised explanations for driving the plate motions. Harold Hess [6] proposed that the mantle was governed by thermal cells: oceanic ridges would be on top of ascending upwellings from the mantle and subduction zones on top of downwellings. Even very simplified, this explanation provided a first scheme of plate motions driven by mantle convection. By definition, mantle convection refers to the heat transported by the large-scale motion of currents in the mantle and corresponds to a complex process.

The mantle is heated from below (heat flow provided mainly by the secular cooling of the core and its radioactivity), from within (radioactivity), cooled from above and cools off with time (secular cooling).

Yet, the way in which lithospheric plates are connected to the underlying mantle is still debated. For example, the contentious 'chicken and egg problem' (does the plate drive the underlying mantle convection or does the mantle drag the plate?) is still put under questions for a few plates (e.g., the Eurasian plate).

\section{MATHEMATICAL DESCRIPTION OF PLATE TECTONICS}

From the angular velocity of plate motions, one is able to compute two scalar fields which are of fundamental importance in hydrodynamic considerations: the horizontal divergence (ridge-trench system) and the radial vorticity (transform fault and plate spin). As the divergence of the velocity is null, the velocity field can be described in a unique way by two complementary and orthogonal scalar fields, namely the poloidal and toroidal scalar fields.

$$
\vec{v}=-1 / \rho_{s} \underbrace{\left(\overrightarrow{\text { rotrot }}\left(\phi \frac{\vec{g}}{g}\right)\right.}_{\text {poloidal part }}+\underbrace{\left.\overrightarrow{\operatorname{rot}}\left(\psi \frac{\vec{g}}{g}\right)\right)}_{\text {toroidal part }}
$$


$\psi$ is called the toroidal field and $\phi$ the poloidal field. The toroidal field describes lateral motions around a vertical axis and the poloidal field depicts vertical motions around a lateral axis. In a spherical reference frame, $\phi$ and $\psi$ can be expressed by spherical harmonics:

$$
\left\{\begin{array}{l}
\psi(r, \lambda, \varphi)=\Sigma_{l, m} T_{l, m}(r) Y_{l, m} \\
\phi(r, \lambda, \varphi)=\Sigma_{l, m} S_{l, m}(r) Y_{l, m}
\end{array}\right.
$$

A simple calculation shows that the poloidal and toroidal scalar fields are directly related to the horizontal divergence and the radial vorticity, respectively [7]:

$$
\overrightarrow{\nabla_{H}} \cdot \vec{v}=-\sum_{l, m} \frac{l(l+1) \dot{S}_{l, m} Y_{l, m}}{\rho r^{2}}
$$

and

$$
(\overrightarrow{\operatorname{rot} v}) \cdot \overrightarrow{e_{r}}=\sum_{l, m} \frac{l(l+1) T_{l, m} Y_{l, m}}{\rho r^{2}}
$$

\section{MANTLE FLOW SIMULATIONS OF THE EARTH}

The simulations are performed using 3D spherical model of mantle convection in which plates are dynamically coupled to the underlying mantle flow. I employ a 3D, spherical-shell, numerical model of time-dependent thermal convection in a mantle which is treated as an incompressible viscous fluid. The dimensionless Boussinesq equations satisfying the principles of conservation of mass, momentum and energy are, respectively:

$$
\begin{gathered}
\nabla \cdot \mathbf{v}=0 \\
-\nabla P_{d}+R a T \hat{\mathbf{r}}+\nabla \cdot \tau=0 \\
\partial T / \partial t+\mathbf{v} \cdot \nabla T=\nabla^{2} T+Q
\end{gathered}
$$

where $\mathbf{v}$ is the velocity vector, $P_{d}$ is the dynamical pressure, $T$ is the temperature field, $\tau$ is the deviatoric stress tensor, $R a$ is the Rayleigh number, and $Q$ is the dimensionless internal heating. We non-dimensionalize the conservation equations according to the relations:

$$
r=r / d, \quad t=t \kappa / d^{2}, \quad v=v d / \kappa \quad \text { and } \quad T=\left(T-T_{t o p}\right) / \Delta T
$$

with

$$
\kappa=610^{-7} \mathrm{~m}^{2} \mathrm{~s}^{-1}, \quad g=10 \mathrm{~ms}^{-2}, \quad \Delta T=2000 \mathrm{~K}, \quad d=2891 \times 10^{3} \mathrm{~m}
$$

and $T_{\text {top }}=0$.

The Rayleigh number appearing in the above momentum conservation equation is defined as:

$$
\frac{\operatorname{ag} d^{3} \rho \Delta T}{\kappa \eta}
$$

The plates are coupled to the mantle using a torque balancing procedure which depends on the flow-induced viscous stresses generated by the internal mantle flow and the flow associated with the surface plates [8,9]. The model developed by Quéré and colleagues is the only 3D spherical code with dynamically coupled plates. This method, which ensures the retrieval (within $\pm 10 \%$ ) of the main global observables at the Earth's surface (mean plate velocity, global heat flux and potential 
temperature under oceanic ridges), is unique and provides new opportunities to tackle questions such as the coupling between plates and the deeper mantle. Due to its parametrization, this method can be used either with imposed plate motions (e.g., past or present-day) or with freely moving plates in response to buoyancy driven mantle flow.

The simulations presented here used three different pre-defined plate geometry: 4 plate geometry, 12 plate geometry and 38 plate geometry (Fig. 2). The model parameters which I vary to provide a fit to the mean surface observables (to within $\pm 10 \%$ ) are the mantle viscosity, the amount of internal
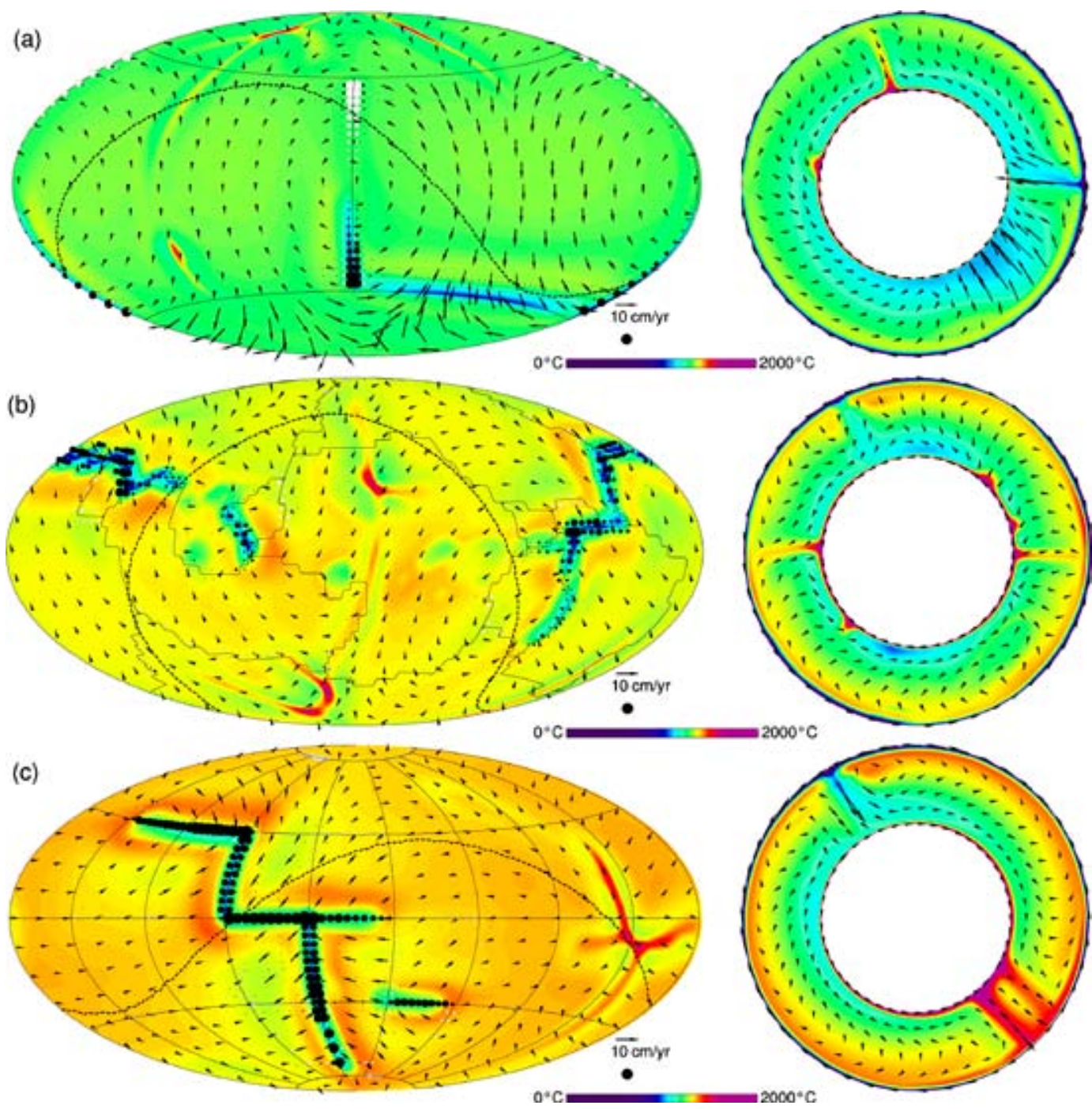

Figure 2: Mantle flow simulations with different plate geometries. The lateral temperature variations at $670 \mathrm{~km}$ depth with the prescribed surface velocity field (left column). Whole-mantle cross-sections, along dashed line (right column). (a) 4 plate model (internal heating $30 \mathrm{TW}$, lower mantle viscosity $10^{22} \mathrm{~Pa} \mathrm{~s}$ ). (b) 12 plate model (present-day geometry; internal heating $28 \mathrm{TW}$, lower mantle viscosity $3 \times 10^{22} \mathrm{~Pa} \mathrm{~s}$ ). (c) 38 plate model (internal heating $30 \mathrm{TW}$, lower mantle viscosity $2.5 \times 10^{22} \mathrm{~Pa} \mathrm{~s}$ ). 
heating and the temperature at the core-mantle boundary. All these models use a viscosity increase of 30 at $670 \mathrm{~km}$ depth.

\section{MYSTERIES OF THE EARTH}

\subsection{Quasi-equipartition between the horizontal divergence and radial vorticity}

Surprisingly, kinetic energy in present-day plate motions is partitioned approximately equally between horizontal divergence (poloidal) and radial vorticity (toroidal) parts (Fig. 3) [10]. I made experiments with different plate geometry on top of the 3D spherical code $(1,2,3,4,12,14,15,28$, 38 and 42 plates). When I plot the degree variance of the poloidal (or horizontal divergence) and toroidal (or radial vorticity) components of the surface velocity field, only a geometry close to the observed present-day geometry shows almost the same toroidal to poloidal ratio that exists at presentday (compare Figs 3 and 4a). With 4 or 38 plates, the toroidal component spectrum is totally different from the poloidal component spectrum (see Fig. $4 \mathrm{~b}$ and c). It suggests that only a geometry close to the one which is observed at present-day can reproduce an equipartition between the poloidal and toroidal components of plate motions (Figs. 3 and 4a).

\subsection{Minimization of the toroidal scalar field}

The observed ratio of toroidal to poloidal components seems to be a minimum as this ratio is less than would be expected for plate motions different from those observed at present-day. This result has been shown through a Monte Carlo simulation of plate motions [12]. The present-day geometry was used and a range of possible plate motions has been investigated through a Monte Carlo simulation. This experiment showed that the observed toroidal:poloidal ratio is less than would be expected for most plates motions, given the present-day geometry of plates. This suggests that the plate mantle convection system tends to minimize the toroidal components of plate motions. This conclusion is supported by studies of convecting systems with plates, where the system evolves to a final state that has the lowest relative toroidal power. The same conclusion

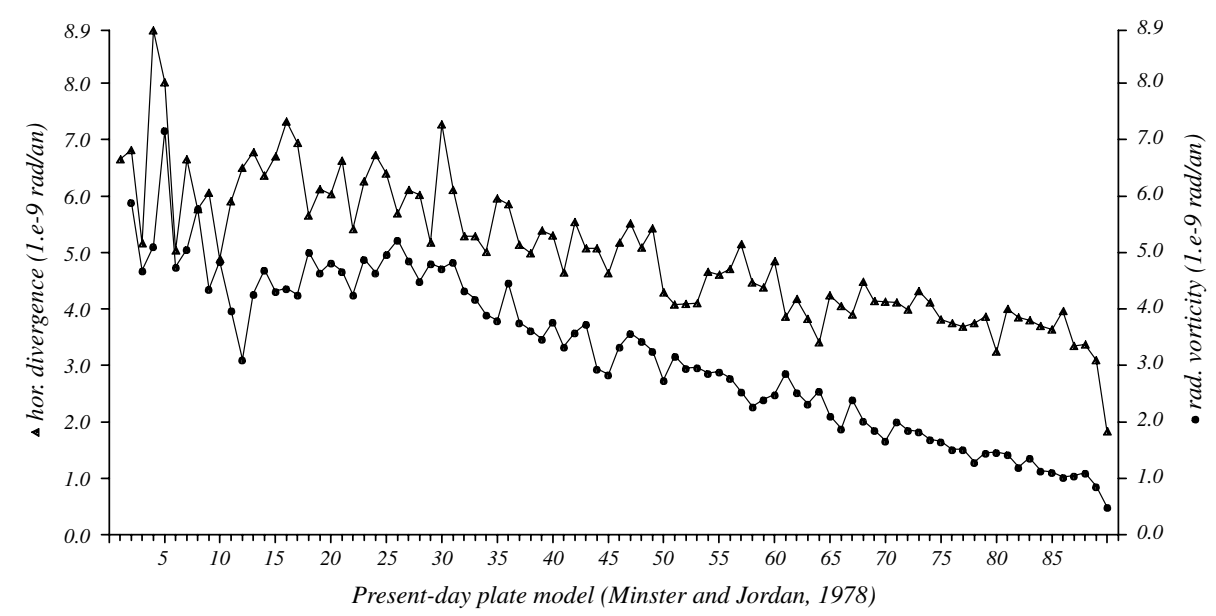

Figure 3: Comparison of horizontal divergence and radial vorticity spectra with the present-day plate motions (AM1-2 model [11]). 

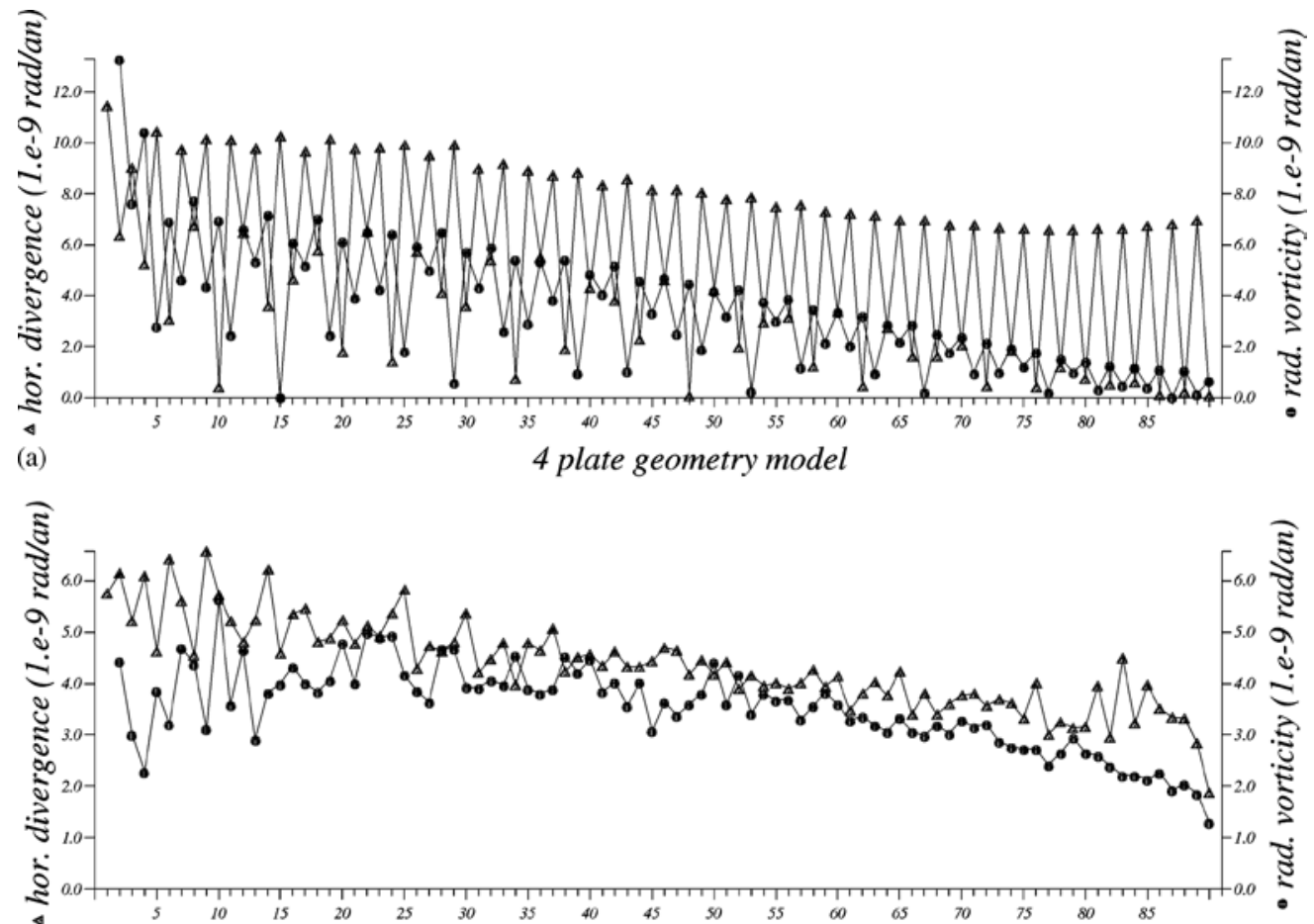

(b)

12 plate geometry model

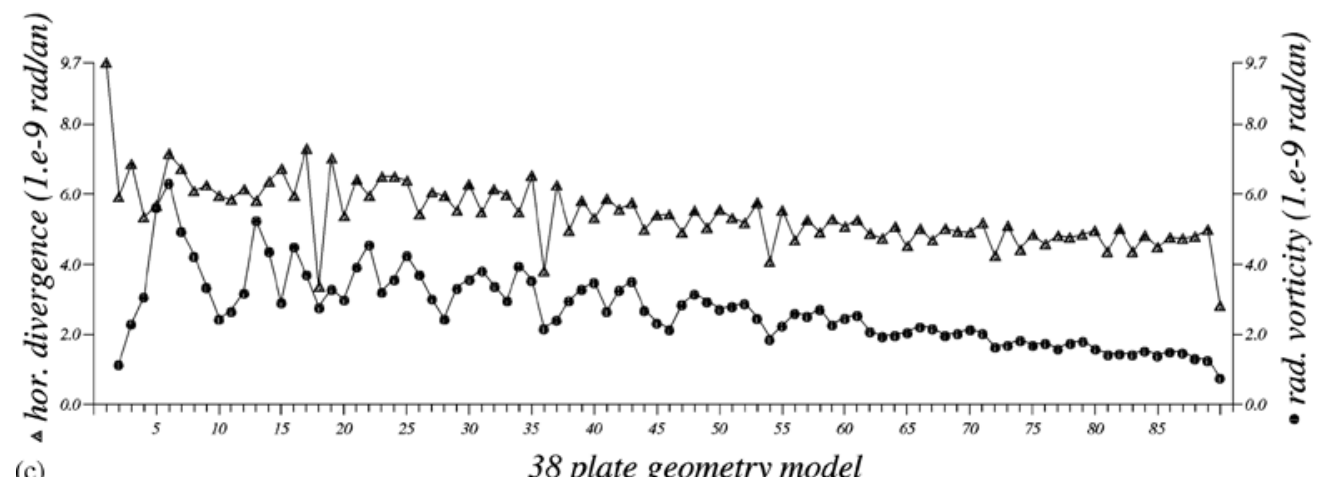

Figure 4: Comparison of horizontal divergence and radial vorticity spectra. (a) 4 plate model. (b) 12 plate model (present-day geometry). (c) 38 plate model.

has been obtained for the Cenozoic (i.e. the period 60-0 Ma) [13] and for the Mesozoic (i.e., the period 120-60 Ma) [14]. The toroidal motion seems to facilitate the efficiency of convective flow [15].

Only the poloidal field is associated with vertical motions, and hence with the liberation of potential energy arising from the vertical motion of density variations in the gravitational field. It is only the poloidal field that is directly excited by body forces; the toroidal field is excited indirectly by the motions of the plates. However, both fields contribute to the dissipation of energy in the system. 
Thus it is plausible (but not yet proven) that the system would evolve to the state that minimizes the viscous dissipation relative to the rate of potential energy release. This could be accomplished by minimizing the toroidal field relative to the poloidal field.

O'Connell et al. [12] presume that, since the toroidal velocities are not involved in the heat transfer, the convective process develops a dynamical structure reducing the loss of kinetic energy, so that the partitioning essentially depends on the plate distribution. By using the constructal theory, we should be able to explain how the most efficient Earth system works with an almost equipartition of kinetic energy which implies a geometry close to the one observed at present-day. In brief, this optimal geometry should facilitate the efficiency of the convective flow knowing its present-day parameters (degree of curvature, viscosity profile, mantle volume, etc.). The constructal theory predicts the main three zones of the global atmospheric circulation, as well as the average speed of atmospheric and oceanic flow and the average temperature on Earth [16]. Plate tectonics are characterized by heat source/heat sink and plate motions represent the heat transport. Mantle convection and atmospheric circulation rely on the same non-linear equations but with different time-scales and parameters. As the average plate velocity and the global heat flow depend on the plate geometry, it is probable that these values could also be predicted through the constructal theory. Shahnas et al. showed that the mean temperature can also be predicted for 3D models of mantle convection [17].

\section{COVARIANCE OF PLATE TECTONICS}

The constructal theory is covariant [18] as the shape is changing in relation to the flow as is plate tectonics. Plate motion reconstructions show that the geometry and plate motions are evolving slowly with time (Fig. 5). The net rotation of the lithosphere (toroidal degree 1) is very low in this new Indo-Atlantic reference frame and appears to be quasi-inexistent at certain periods [19].

Contrary to what has been previously thought in the past, during the Cenozoic period, no major plate reorganization happened. The bend observed in the Hawaiian volcano track $43 \mathrm{Ma}$, which was previously explained by a drastic change in direction of the Pacific plate, is due to the motion of the Hawaiian hotspot that is moving [20]. The previous interpretation (plate reorganization) was made on the assumption of the fixity of the Pacific hotspots which has recently been placed into considerable doubt and they appear, especially the Hawaiian hotspot, to be subject to significant drifting.

\section{MANTLE FLOW SIMULATION DURING THE PAST 120 MILLION YEARS}

I use plate motion reconstructions established mainly by paleomagnetic data on top of my convective model during the Cenozoic and Mesozoic periods (i.e., the last 120 million years). The Mesozoic period used the plate motion reconstruction provided by Lithgow-Berthelloni and Richards [21] and the Cenozoic period used plate motions presented by Quéré et al. [19]. After having spanned 120 million years of Earth's history, the simulation at present-day (0 Ma) presents a broad agreement between the location of the hot and cold regions in the convection model and the similarly configured regions of hotter and colder than average mantle in the tomography model by Simmons et al. [22] (compare Figs. 6 and 7). The five mantle upwellings appear to be located near the present-day locations of the Cape Verde, Crozet, Easter, Azores and Caroline hotspot volcanoes (Fig. 7). This correlation between completely independent data sets means that a $3 \mathrm{D}$ convective model combined with a plate motion reconstruction is able to reproduce the main upwellings/ downwellings observed on Earth. 
(a) $60 \mathrm{Ma}, \mathrm{v}=4.59 \mathrm{~cm} / \mathrm{yr}$

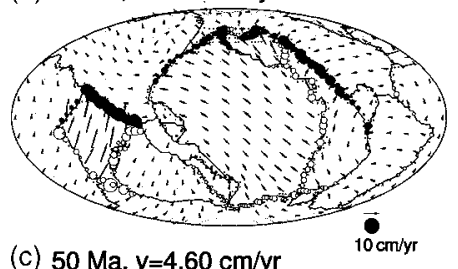

(c) $50 \mathrm{Ma}, \mathrm{v}=4.60 \mathrm{~cm} / \mathrm{yr}$

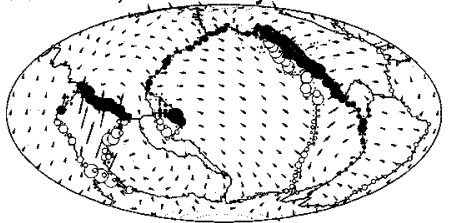

(e) $40 \mathrm{Ma}, \mathrm{v}=4.34 \mathrm{~cm} / \mathrm{yr}$

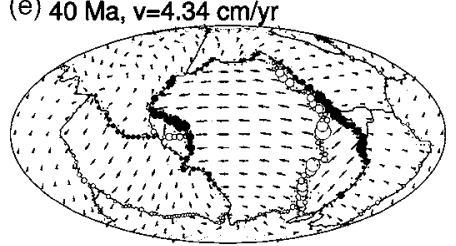

(g) $30 \mathrm{Ma}, \mathrm{v}=4.90 \mathrm{~cm} / \mathrm{yr}$

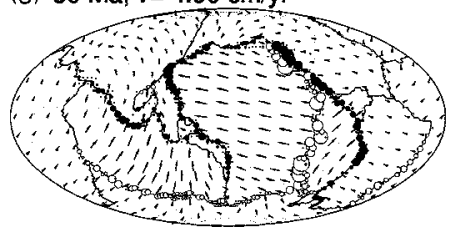

(i) $20 \mathrm{Ma}, \mathrm{v}=4.99 \mathrm{~cm} / \mathrm{yr}$

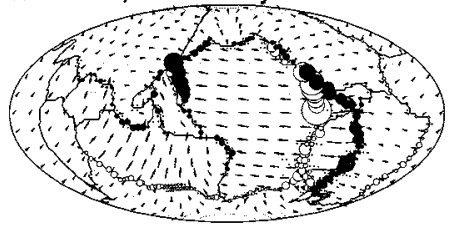

(k) $10 \mathrm{Ma}, \mathrm{v}=4.53 \mathrm{~cm} / \mathrm{yr}$

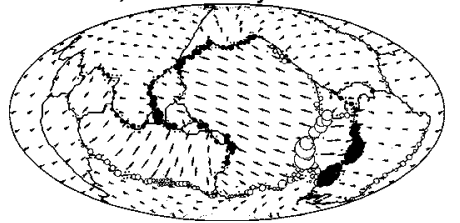

(b) $55 \mathrm{Ma}, \mathrm{v}=4.87 \mathrm{~cm} / \mathrm{yr}$

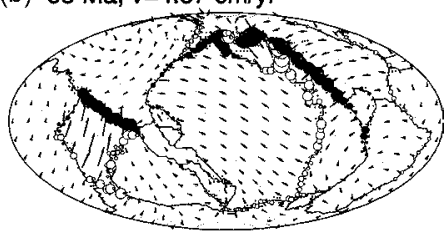

(d) $45 \mathrm{Ma}, \mathrm{v}=3.69 \mathrm{~cm} / \mathrm{yr}$

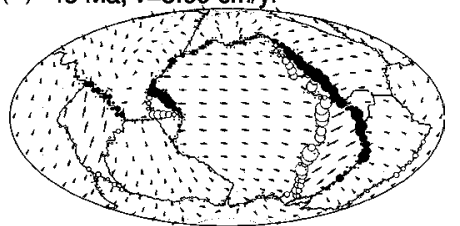

(f) $35 \mathrm{Ma}, \mathrm{v}=4.41 \mathrm{~cm} / \mathrm{yr}$

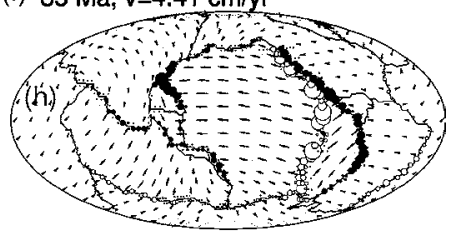

(h) $25 \mathrm{Ma}, \mathrm{v}=4.80 \mathrm{~cm} / \mathrm{yr}$

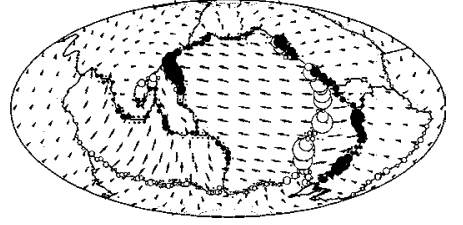

(j) $15 \mathrm{Ma}, \mathrm{v}=4.51 \mathrm{~cm} / \mathrm{yr}$

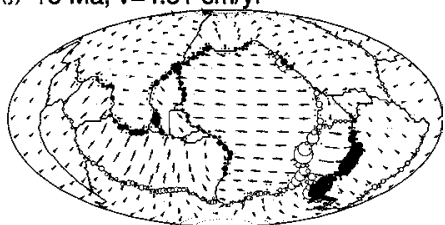

(l) $05 \mathrm{Ma}, \mathrm{v}=4.61 \mathrm{~cm} / \mathrm{yr}$

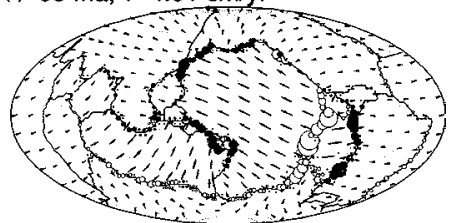

Figure 5: Observed velocities in the no-net-rotation reference frame: (a) $60 \mathrm{Ma}$, (b) $55 \mathrm{Ma}$, (c) $50 \mathrm{Ma}$, (d) $45 \mathrm{Ma}$, (e) $40 \mathrm{Ma}$, (f) $35 \mathrm{Ma}$, (g) $30 \mathrm{Ma}$, (h) $25 \mathrm{Ma}$, (i) $20 \mathrm{Ma}$, (j) $15 \mathrm{Ma}$, (k) $10 \mathrm{Ma}$ and (l) $5 \mathrm{Ma}$.

\section{CONCLUSIONS}

Plate tectonics is not an 'chicken and egg problem'. The lithoshere which describes the rigid tectonic plates corresponds to the upper boundary layer of the convection system. Within this vision, the lithosphere and the mantle are coupled and source or consequence cannot be dissociated 

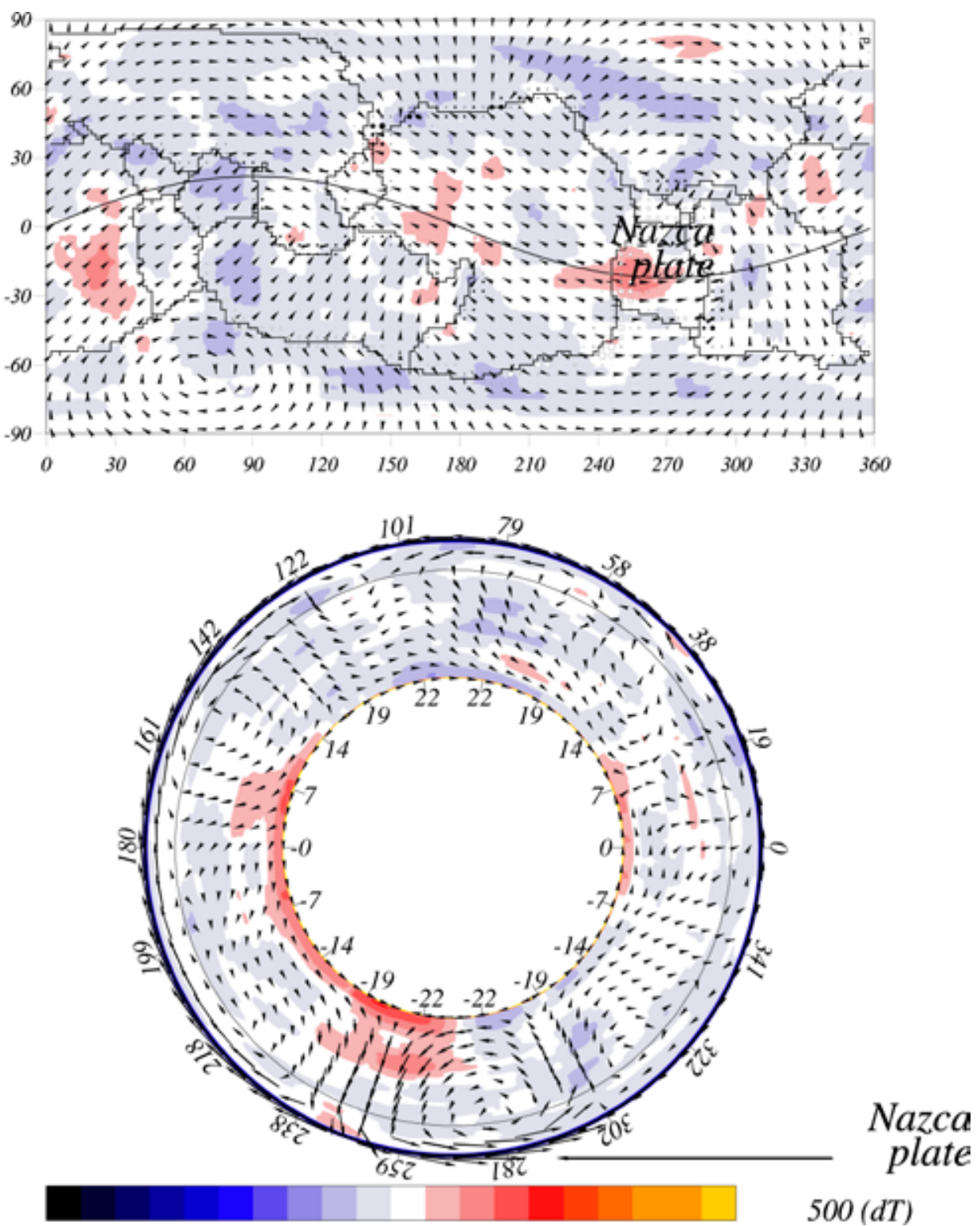

Figure 6: Temperature anomalies at $1900 \mathrm{~km}$ depth obtained from seismic shear wave anomalies in the Simmons et al. model [22]. The tomographic-induced velocity field and corresponding temperature field inside the mantle are drawn along the black line. The fastest convective cell is under the Nazca plate.

as they are part of the same system. The plates correspond to the upper plate boundary and participate in the convective system. Due to the plate-mantle coupling, plate motions match the mantle flow below and vice versa, the mantle flow reflects the plate motions above. The present-day geometry is an optimal geometry that matches the Earth's convective system (mantle volume, 

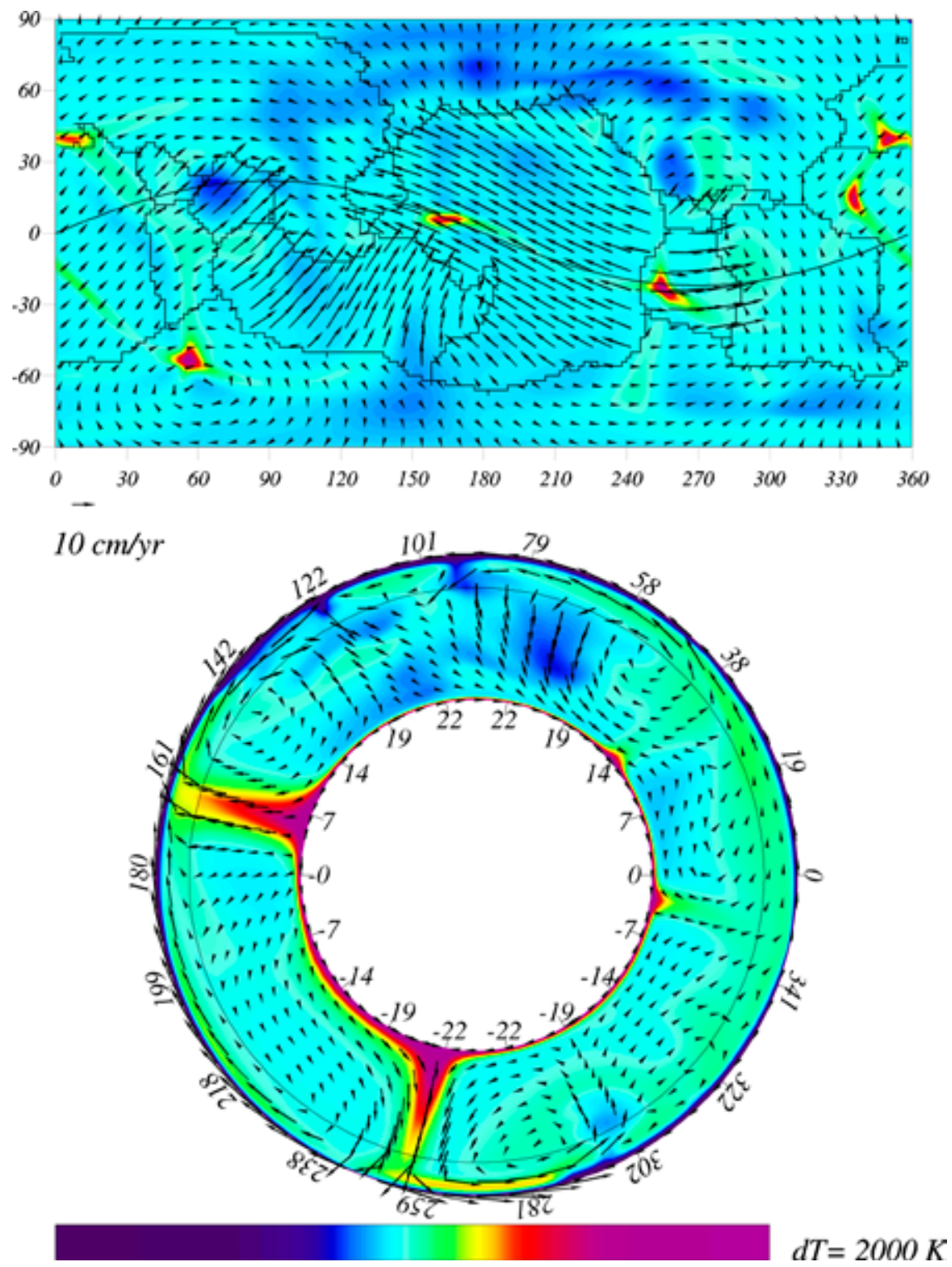

Figure 7: Lateral temperature variations at $1900 \mathrm{~km}$ depth and surface velocity field. The five predicted 'hotspot'-like mantle plumes are Cape Verde, Crozet, Easter, Azores and Caroline hotspots. The convective-induced velocity field and corresponding temperature field inside the mantle are drawn along the black line. [Note the cross-sections of Figs. 6 and 7 display equivalent upwellings and downwellings, respectively, and that the cell under the Nazca plate is the fastest convective cell].

degree of curvature, viscosity profile, amount of internal heating, temperature at the base of the mantle) which provides an equipartition between the poloidal and toroidal components through a possible minimization of toroidal energy. Constructal theory predicts that the shape must evolve through time to provide an easier flow access through minimization of imperfections. 
Plate motions seem to evolve by developing a convective process that reduces the loss of kinetic energy, so that the partitioning essentially depends on the plate distribution and minimizes the toroidal flow. The constructal theory is covariant as is plate tectonics which depicts plate motions and geometry which are evolving slowly in time in coherence with the underlying mantle flow.

\section{REFERENCES}

[1] Wegener, A., Die Entstehung der Kontinente, Petermanns Mitteilungen, 1912.

[2] Holmes A., Radioactivity and earth movements. Trans. Geol. Soc. Glasgow, 18, pp. 559-606, 1931.

[3] Morgan, W.J., Rises, trenches, great faults, and crustal blocks. J. Geophys. Res., 73, pp. 1959-1982, 1968. doi:10.1029/JB073i006p01959

[4] McKenzie, D.P. \& Parker, R.L., The north Pacific: an example of tectonics on a sphere. Nature, 216, pp. 1276-1279, 1967. doi:10.1038/2161276a0

[5] Argus, D. \& Gordon R., No net rotation model of current plate velocities incorporating plate motion model NUVEL-1. Geophys. Res. Lett., 18, pp. 2039-2042, 1991. doi:10.1029/91GL01532

[6] Hess, H., History of ocean basins. Petrologic Studies: a Volume in Honor of A.F. Buddington, eds A.E.J. Engel et al., Geophysical Society of America: Boulder, Colorado, 1962.

[7] Forte, A.M. \& Peltier, W.R., Plate tectonics and aspherical Earth structure: the importance of poloidal-toroidal coupling. J. Geophys. Res., 92, pp. 3645-3679, 1987. doi:10.1029/ JB092iB05p03645

[8] Monnereau, M. \& Quéré, S., Spherical shell models of mantle convection with tectonic plates. Earth planet. Sci. Lett., 184, pp. 575-587, 2001. doi:10.1016/S0012-821X(00)00334-4

[9] Quéré, S. \& Forte, A.M., Influence of past and present-day plate motions on spherical models of mantle convection: implications for mantle plumes and hotspots. Geo-phys. J. Int., 165, pp. 1041-1057, 2006. doi:10.1111/j.1365-246X.2006.02990.x

[10] Hager, B.H. \& O'Connell, R.J., Subduction zone dip angles and flow driven in the earth's mantle. Tectonophysics, 50, pp. 111-133, 1978. doi:10.1016/0040-1951(78)90130-0

[11] Minster, J.B. \& Jordan, T.H., Present-day plate motions. J. Geophys. Res., 83, pp. 5331-5354, 1978. doi:10.1029/JB083iB11p05331

[12] O'Connell, R.J., Gable, C.W. \& Hager, B.H., Toroidal-poloidal partitioning of lithospheric plate motions. Glacial Isostasy, Sea Level and mantle Rheology, eds R. Sabadini \& K. Lambeck, Kluwer Academic Publishers: Dordrecht, 1991.

[13] Cadek, O. \& Ricard, Y., Toroidal/poloidal energy partitioning and global lihospheric rotation during Cenozoic time. Earth Planet. Sci. Lett., 109, pp. 621-632, 1992. doi:10.1016/0012821X(92)90120-K

[14] Lithgow-Bertelloni, C., Richards, M.A., Ricard, Y., O'Connell, R.J.\& Engebretson, D.C., Toroidal-poloidal partitioning of plate motion since 120 Ma, Geophys. Res. Lett., 20, pp. 357-378, 1993. doi:10.1029/93GL00168

[15] Bercovici, D., A simple model of plate generation from mantle flow. Geophys. J. Int., 114, pp. 635-650, 1993. doi:10.1111/j.1365-246X.1993.tb06993.x

[16] Reis, A.H., Bejan A., Constructal theory of global circulation and climate, Int. J. Heat Mass. Trans., 49(11-12), pp. 1857-1875, 2006. doi:10.1016/j.ijheatmasstransfer.2005.10.037

[17] Shahnas, M.H., Lowman, J.P., Jarvis, G.T. \& Bunge, H.P., Convection in a spherical shell heated by an isothermal core and internal sources: implications for the thermal state of planetary mantles. Phys. Earth Planet. Int., 168, pp. 6-15, 2008. doi:10.1016/j.pepi.2008.04.007 
[18] Bejan, A., Shape and Structure, from Engineering to Nature, Cambridge University Press: Cambridge, UK, 324 p., ISBN 0521793882, 2000.

[19] Quéré, S., Rowley, D., Forte, A. \& Moucha, R., 'New Insights on Cenozoic Plate Evolution and Mantle Dynamics in the Indo-Atlantic Hotspot Reference Frame', Eos Trans. AGU, Fall Meeting Suppl., Abstract DI53A-1681, 2008.

[20] Tarduno, J.A., Duncan, R.A., Scholl, D.W., Cottrell, R.D., Steinberger, B., Thordarson, T., Kerr, B.C., Neal, C.R., Frey, F.A., Torii, M., Carvallo, C., The Emperor seamounts: southward motion of the Hawaiian hotspot plume in Earth's mantle. Science, 301, pp. 1064-1069, 2003. doi: $10.1126 /$ science. 1086442

[21] Lithgow-Bertelloni, C. \& Richards M., The dynamics of Cenozoic and Mesozoic plate motions. Rev. Geophys., 36, pp. 27-78, 1998. doi:10.1029/97RG02282

[22] Simmons, N.A., Forte, A.M., Grand, S.P., 2007, Thermochemical structure and dynamics of the African superplume. Geophys. Res. Lett., 34, L02301. doi:10.1029/2006GL028009 